\title{
Actitudes e Intereses hacia las TIC en una Muestra de Estudiantes de Posgrado
}

\section{Attitudes and Interests in ICTs in a Sample of Postgraduate Students}

\author{
Francisco Manuel Morales Rodríguez \\ Universidad de Málaga, España
}

\begin{abstract}
Resumen. El objetivo del presente trabajo es aportar una evaluación de actitudes e intereses hacia las TIC en una muestra de 224 estudiantes del Máster en Profesorado de Educación Secundaria Obligatoria y Bachillerato, Formación Profesional y Enseñanza de Idiomas de la Universidad de Málaga, con edades comprendidas entre 22 y 49 años. Las cuestiones que refleja el cuestionario hacen referencia a los conocimientos que se tienen a nivel informático, hasta qué punto consideran útil Internet, desde dónde suelen acceder, con qué frecuencia lo hacen y si consideran útil en su proceso interactivo de aprendizaje la utilización del visionado de vídeos, Power point, plataforma Moodle, foros, wikis, chats, videoforums y blogs, así como numerosas cuestiones sobre cuáles son las funciones que Internet puede llegar a tener en el contexto educativo universitario. Los datos demuestran que existen diferencias en la percepción de las funciones que las TIC pueden tener en dicho contexto según el género y la especialidad.
\end{abstract}

Palabras clave: actitudes, TIC, aprendizaje, estudiantes de posgrado.

\begin{abstract}
The aim of this study is to provide an assessment of attitudes and interests with regard to Information and Communication Technology (ICTs) in a sample of 224 post-graduate students of the MSc in Secondary Education, aged between 22 and 49. The questions posed in the questionnaire evaluate the important aspects of interactive learning: knowledge of computers, knowing the value of using the internet, how this is generally accessed, the frequency of use given to it and whether they consider the use of video, PowerPoint, Moodle, forums, wikis, chats, Video Forums and blogs to be useful in their interactive learning, as well as a series of questions about the functions that the internet could have in the educational context. The data show that there are differences in perception of the role that ICTs may play in this context according to gender and specialty.
\end{abstract}

Keywords: attitudes, ICT, learning, postgraduate students.

\section{Introducción}

En la actualidad existen factores tecnológicos, culturales, demográficos, políticos y económicos que han cambiado las reglas del juego en las organizaciones e instituciones del siglo XXI. Uno de dichos cambios más significativos que pueden señalarse es cómo el acceso masivo a las Tecnologías de la Información y de la Comunicación (TIC) está cambiando nuestra concepción del tiempo y transformando las mismas.

El desarrollo tecnológico que están experimentando nuestras sociedades junto con una política económica favorable a la integración de las economías es un elemento determinante de la globalización (así como de sus costes y beneficios). El progreso tecnológico e innovador de algún modo revierte en un mundo más pequeño con un intercambio de bienes más barato y menos costoso. La cultura europea ha tratado de mantener tanto la competitividad como la cohesión social sin perder de vista los principios del Estado del

La correspondencia sobre este artículo debe enviarse al e-mail: framorrod@uma.es
Bienestar, en esa búsqueda continua por mantener el necesario equilibrio entre la solidaridad y la eficiencia. La innovación se ha convertido en una auténtica necesidad y el factor humano que componen las organizaciones en una garantía de supervivencia, desarrollo, progreso social y crecimiento.

El Nuevo Modelo de Educación Superior trae consigo nuevas metodologías, demandas y retos, donde el uso de las TIC, especialmente Internet, como herramienta didáctica será indispensable en el proceso de enseñanza/aprendizaje. Son muchas las ventajas y aportaciones que las tecnologías digitales pueden tener para el mundo de la educación en la actualidad. No obstante, aún en algunos ámbitos del quehacer humano la utilización de las TIC parece generar, cuando menos, controversia y un excesivo grado de suspicacia. Resulta obvio que la utilización de estos medios puede tener tanto ventajas como inconvenientes (Cabero y Gisbert, 2005; García-Valcárcel, 2007; Sangrá y González, 2004) y de ahí la necesidad de tratar de identificar qué tipo de tecnologías se pueden usar con fines educativos con vistas a las competencias que el alumnado ha de adquirir según el Espacio Europeo de Educación Superior. Entre los inconve- 
nientes, según plantea Carr (2011), pueden estar el hecho de su potencialidad para disminuir la capacidad de concentración y reflexión, es decir, se puede buscar y compartir información de forma rápida e inmediata pero también se puede perder capacidad para la lectura y para mantener una línea de pensamiento profundo en cualquier materia o aspecto durante cierto tiempo. En cambio, son numerosos los estudios emergentes (Barroso y Cabero, 2010; Cebrián y Gallego, 2011; De Pablos, Area, Valverde y Correa, 2010; centrados en los procesos educativos con TIC en la sociedad del conocimiento y sus aplicaciones al mundo educativo, en los que se destacan, por ejemplo, las siguientes ventajas: posibilidad de asumir diferentes modelos de enseñanza/aprendizaje más innovadores, interactivos y adaptados para diferentes tipos de estudiantes, aparición de las páginas web de las asignaturas, difusión de la información a una innumerable cantidad de personas e instituciones del mundo, aumento de las posibilidades de interacción entre los diferentes actores educativos como son el profesorado y el alumnado, papel más activo del alumnado, optimización del aprendizaje significativo, generación de nuevos canales de comunicación favoreciendo la aparición de listas de distribución, grupos de trabajo y foros de discusión, elaboración de material docente de forma colaborativa, etc.

La Declaración de Bolonia supone el inicio del proceso de construcción de este espacio que responde a la iniciativa de la Unión Europea de crear un sistema homogéneo de educación en este nivel, que fomente el desarrollo de todos los miembros, movilidad, empleabilidad y capacidad de innovación de los ciudadanos (Rubio y Álvarez, 2010). En este sentido, el Real Decreto 55/2005 establece la nueva estructura de los estudios oficiales surgiendo las enseñanzas de Grado en las Universidades donde se plantea de forma explícita que las mismas deben dar respuesta a las necesidades formativas de futuro incorporando las TIC en el proceso de aprendizaje a través de metodologías didácticas innovadoras. Los planes de estudio deben analizar y evaluar de forma más precisa actitudes hacia dichas metodologías por parte de alumnado, profesorado e instituciones de forma que contribuyan a facilitar los procesos de aprendizaje virtuales a lo largo de la vida y tratando de configurar un proceso continuo de innovación que incorpore las mejores prácticas educativas internacionales y las aplicaciones tecnológicas más avanzadas.

En pleno proceso de convergencia europea, es necesario preparar a los jóvenes en competencias para que puedan desenvolverse profesionalmente de forma exitosa, con alta cualificación para orientar el progreso económico, intelectual y cultural que necesitan nuestras sociedades. Para fomentar la adquisición de estas competencias son necesarias las metodologías participativas y colaborativas que nos ofrece la Web 2.0. En esta línea, Fernández y Mena (2010) conside- ran que el papel del profesor y la concepción que éste tiene de la enseñanza también ha cambiado y los profesionales deben desarrollar nuevas competencias, personales y profesionales como el uso de herramientas TIC, iniciativa, creatividad, trabajo en equipo, estrategias de resolución de problemas para crear el conocimiento preciso que les permita afrontar con éxito los nuevos retos. Es necesario asumir un nuevo papel de mediador que fomente tanto el trabajo en grupo o colaborativo así como también el pensamiento autónomo o crítico.

Otros autores (Ríos y Ruiz, 2011) también consideran que las TIC son imprescindibles para afrontar muchas de las dificultades y problemas existentes en la sociedad y se requieren de evaluaciones continuas de los elementos que conforman las competencias tales como actitudes, habilidades, conocimientos y valores tratando de implicar a los estudiantes, docentes y autoridades universitarias en la implementación de un modelo educativo basado en competencias. Resulta prioritario en este modelo que los estudiantes que forman parte de las instituciones de enseñanza superior y que son los auténticos protagonistas del proceso de enseñanza/aprendizaje se sientan apoyados para poder garantizar su éxito y satisfacción profesional en el ámbito universitario y productivo. Ello justifica la necesidad de evaluaciones más precisas, como se hace en el presente estudio, de cuáles son sus actitudes e intereses hacia las TIC como herramientas de aprendizaje y de comunicación.

En el ámbito universitario en algunos estudios (De Pablos, 2009; Trujillo, 2005) se viene señalando la necesidad de no usar de forma indiscriminada las TIC sin reflexionar sobre la introducción que dichos cambios suponen considerando aspectos como su grado de utilidad y adaptabilidad. En este sentido, uno de los elementos importantes en el proceso de integración de las TIC en la Universidad es conocer precisamente las opiniones y actitudes del alumnado respecto al uso de las TIC en su proceso de aprendizaje (Martínez y Aguaded, 2004).

Por todo lo dicho, el objetivo del presente trabajo es aportar una evaluación de actitudes e intereses hacia las TIC en una muestra de 224 estudiantes de posgrado pertenecientes al Máster en Profesorado de Educación Secundaria Obligatoria y Bachillerato, Formación Profesional y Enseñanza de Idiomas correspondiente a las especialidades de Lengua Extranjera (Inglesa y Francesa); Literatura, Latín y Griego; y Tecnología, Informática y Procesos Industriales, analizando diferencias en función del género y de la especialidad.

Estos datos permiten concluir respecto a la importancia del uso de las TIC en el proceso de enseñanza/aprendizaje así como las posibles ventajas e inconvenientes que tiene su utilización tanto por parte del profesorado como por el alumnado de posgrado en el reciente Máster de Educación Secundaria. 


\section{Método}

\section{Participantes}

Los participantes han sido 224 estudiantes, con edades comprendidas entre 22 y 49 años, pertenecientes al Máster en Profesorado de Educación Secundaria Obligatoria y Bachillerato, Formación Profesional y Enseñanza de Idiomas de la Universidad de Málaga siendo 64 correspondientes a las especialidad de Lengua Extranjera Inglés y Francés; 58 de la especialidad Lengua, Literatura, Latín y Griego; y 102 de dos cursos (mañana y tarde) de la especialidad de TIPI (Tecnología, Informática y Procesos Industriales). Todos respondieron al cuestionario en una aplicación colectiva en el grupo clase.

\section{Instrumentos}

Cuestionario de Actitudes e Intereses hacia las TIC (Morales, 2009); además de las instrucciones pertinentes para la cumplimentación de dicho cuestionario. Como se ha señalado, las cuestiones presentes en el cuestionario hacen referencia a los conocimientos que se poseen a nivel informático, la frecuencia y el lugar desde donde suelen acceder a Internet y hasta qué punto consideran útil la utilización del visionado de vídeos, Power point, plataforma Moodle, foros, wikis, chats, videoforums y blogs en su proceso de aprendizaje así como cuestiones relacionadas con las posibles funciones y aplicaciones de Internet en el contexto educativo universitario. El formato del cuestionario consiste en una serie de preguntas en las que se pide a los participantes que completen o rodeen la opción que se corresponde con su respuesta. Para conocer las funciones que Internet puede tener en el contexto educativo se ha presentado al propio alumnado una escala tipo Likert para que contesten en qué grado están de acuerdo con una serie de afirmaciones siendo $0=$ nada; $1=$ poco, 2 = bastante, $3=$ mucho. Este cuestionario presenta adecuadas propiedades psicométricas.

\section{Procedimiento}

Los participantes han realizado el cuestionario de forma voluntaria y sus datos han sido introducidos en el SPSS para su posterior análisis. Se llevó a cabo la administración del instrumento de evaluación a los participantes de forma colectiva en hora de clase, según fecha y hora determinada por el calendario académico de la Universidad. La administración del cuestionario fue llevada a cabo por psicólogos expertos en el campo.

\section{Análisis de datos}

La codificación y análisis de datos se hizo con el paquete estadístico SPSS 15.0. Además de realizar análisis descriptivos reflejándose porcentajes, medias y desviaciones típicas, se realizó la prueba $\mathrm{T}$ de Student para muestras independientes para ver si existen diferencias estadísticamente significativas en función del género y de la especialidad concreta del Máster en Profesorado de Educación Secundaria Obligatoria y Bachillerato, Formación Profesional y Enseñanza de Idiomas. Concretamente en este estudio se comparan resultados respecto a las actitudes e intereses hacia las TIC entre las especialidades Lengua Extranjera Inglés y Francés; Lengua, Literatura, Latín y Griego; y la de Tecnología, Informática y Procesos Industriales. Es importante señalar que solo se presentan las tablas con análisis comparativos de los resultados en los que existen diferencias estadísticamente significativas.

\section{Resultados}

En un primer apartado se presentan los resultados de la muestra de estudiantes de posgrado con respecto a sus actitudes e intereses hacia Internet (Tabla 1) así como cuáles son sus conocimientos generales a nivel informático (Tabla 2). Asimismo, se presentan también en este apartado el grado de acuerdo sobre cuáles son las funciones de las TIC en el contexto educativo universitario (Tabla 3) según manifiestan los estudiantes de posgrado del reciente Máster en Profesorado de Educación Secundaria Obligatoria y Bachillerato, Formación Profesional y Enseñanza de Idiomas de la Universidad de Málaga, en los que se hace necesaria una evaluación precisa de dichas actitudes, como se contempla en la organización del mismo y como se presenta en este estudio.

En un segundo apartado, se presentan solo los análisis estadísticamente significativos de diferencia de medias en función del género y de la especialidad en lo que respecta a las funciones de las TIC en el contexto educativo universitario, tras realizar la prueba TStudent para muestras independientes (véase Tablas 4 y 5).

Podemos ver en la Tabla 1 que todo el alumnado conoce Internet (100\%). Así mismo, ante la pregunta de ¿Cómo lo conocieron? El 39.02\% de los participantes afirman que lo conocieron a través de sus amigos, el $21.95 \%$ a través del colegio y el resto a través de la familia, de la televisión u otros medios. Todos los participantes disponen de ordenador personal, la mayor parte de ellos suelen acceder desde casa $(48.44 \%)$ con una frecuencia de una o más veces al día y no disponen de página web. Los servicios más usados de Internet cuando se accede a la red son por orden de uso: correo electrónico (26.89\%); buscadores $(26.89 \%)$; foros $(21.85 \%)$; chats $(12.60 \%) \mathrm{u}$ otros servicios como redes sociales $(11.76 \%)$. El $100 \%$ del alumnado considera que Internet puede crear adicción. El 100\% de los participantes en este estudio consideran que Internet pude 
Tabla 1. Actitudes e intereses hacia Internet en estudiantes del posgrado del Máster de Educación Secundaria (\%)

\begin{tabular}{|c|c|c|}
\hline \multirow[t]{2}{*}{ ¿Sabes qué es Internet? } & No & $0 \%$ \\
\hline & Sí & $100 \%$ \\
\hline \multirow[t]{5}{*}{ Lo conociste a través de: } & Amigos & $39 \%$ \\
\hline & Colegio & $22 \%$ \\
\hline & Familia & $24 \%$ \\
\hline & Televisión & $2 \%$ \\
\hline & Otros medios & $12 \%$ \\
\hline \multirow[t]{2}{*}{ ¿Dispones de ordenador? } & Sí & $100 \%$ \\
\hline & No & $0 \%$ \\
\hline \multirow[t]{5}{*}{ Accedes desde: } & Instituto & $3.12 \%$ \\
\hline & Facultad & $32.81 \%$ \\
\hline & Cibercafé & $3.12 \%$ \\
\hline & Casa & $48.44 \%$ \\
\hline & Trabajo u otros & $12.50 \%$ \\
\hline \multirow[t]{2}{*}{ Frecuencia en que lo usas: } & 1 ó más veces/día & $97 \%$ \\
\hline & 1 ó más veces/semana & $3 \%$ \\
\hline \multirow[t]{5}{*}{ Servicios: } & Correo electrónico & $26.89 \%$ \\
\hline & Chat & $12.60 \%$ \\
\hline & Foros & $21.85 \%$ \\
\hline & Buscadores & $26.89 \%$ \\
\hline & Otros (redes sociales. etc) & $11.76 \%$ \\
\hline \multirow[t]{2}{*}{ Tiene página web: } & Sí & $21.87 \%$ \\
\hline & No & $78.12 \%$ \\
\hline \multirow[t]{2}{*}{ ¿Internet puede crear adicción? } & Sí & $100 \%$ \\
\hline & No & $0 \%$ \\
\hline \multirow[t]{2}{*}{ ¿Se puede utilizar como recurso didáctico? } & Sí & $100 \%$ \\
\hline & No & $0 \%$ \\
\hline
\end{tabular}

Tabla 2. Conocimientos generales a nivel informático

\begin{tabular}{lccc}
\hline & \multicolumn{3}{c}{ \% que consideran nivel } \\
\hline & No poseen & Nivel usuario & Alto \\
\hline Procesadores de Texto & 0 & 36.67 & 63.33 \\
Bases de datos & 25.8 & 51.61 & 22.58 \\
Hojas de cálculo & 19.35 & 64.52 & 16.13 \\
Internet & 0 & 27.59 & 72.41 \\
Diseño gráfico & 47.05 & 44.12 & 8.82 \\
Animación & 55.55 & 27.77 & 16.67 \\
Programación & 74.19 & 12.9 & 12.9 \\
\hline
\end{tabular}

ser utilizado como recurso didáctico. Con respecto al grado en que les gusta Internet el alumnado ha otorgado una puntuación media de 9.21 (en una escala de 010).

Según afirman dichos participantes, en términos generales, sus conocimientos de informática son a nivel de usuario en cuanto a bases de datos y hojas de cálculo, mientras que afirman poseer menos conocimientos sobre programación. Los datos resumidos con los porcentajes correspondientes sobre dichos conocimientos se presentan en la Tabla 2.

A continuación se presentan los resultados más significativos sobre las funciones de las TIC en el contexto educativo obtenidos a partir de la escala tipo likert sobre el grado de acuerdo con ciertas afirmaciones (ver Tabla 3) por parte de todos los estudiantes de la presente muestra del Máster de Educación Secundaria.
Con respecto a las diferencias significativas estadísticamente en función del género en la variable percepción de las funciones de las TIC en el contexto educativo universitario (Tabla 4) en la especialidad Lengua Extranjera del Máster en Profesorado de Educación Secundaria Obligatoria y Bachillerato, Formación Profesional y Enseñanza de Idiomas puede destacarse que las mujeres obtienen medias superiores en el ítem referido a que el nivel socioeconómico de las familias influye en que conozcamos Internet; mientras que los hombres consideran en mayor media en comparación con las mujeres que los weblogs facilitan la comunicación entre el alumnado y con el profesorado.

Finalmente en la tabla 5 se muestran los resultados más significativos en la percepción y predisposición hacia las TIC en función de la especialidad.

Respecto a la especialidad, los resultados demuestran que no existen diferencias estadísticamente significativas en la predisposición hacia las TIC entre el alumnado de la especialidad de Lengua Extranjera y el de la especialidad Tecnología, Informática y Procesos Industriales. Sin embargo, se encuentran diferencias significativas en la percepción y predisposición hacia las TIC entre el alumnado de la especialidad de Lengua Extranjera y la especialidad de Lengua, Literatura, Latín y Griego cuyos resultados se muestran en la Tabla 5. Concretamente, en términos generales, el alumnado de la especialidad Lengua Extranjera muestra mayor predisposición y actitudes 
Tabla 3. Funciones de las TIC en el contexto educativo universitario (\%)

\begin{tabular}{|c|c|c|c|c|}
\hline & Nada & Poco & Bastante & Mucho \\
\hline 1. Internet se puede usar para enseñar & 0 & 0 & 32.5 & 67.5 \\
\hline 2. Internet puede sustituir la función del profesor & 44.74 & 44.74 & 10.53 & 0 \\
\hline 3. Internet sólo saben utilizarlo los alumnos con mayor nivel intelectual & 65.90 & 34.09 & 0 & 0 \\
\hline 4. Internet puede sustituir la función de los libros y las pizarras & 10 & 30 & 47.5 & 12.5 \\
\hline 5. Internet puede compaginarse con las explicaciones que el profesor realiza en pizarra & 3.12 & 3.12 & 25 & 68.75 \\
\hline 6. Sería más útil, divertido y provechoso aprender a través de Internet & 0 & 29.73 & 43.24 & 27.02 \\
\hline 7. Se transmiten valores positivos en Internet & 0 & 35.13 & 54.05 & 10.81 \\
\hline 8. Internet solo puede utilizarse actualmente en zonas socioeconómicamente favorecidas & 26.47 & 47.06 & 23.53 & 2.94 \\
\hline 9. El nivel socioeconómico de las familias influye en que conozcamos Internet & 8.82 & 50 & 32.35 & 8.82 \\
\hline 10. Internet favorece la capacidad para relacionarse con los demás & 5.40 & 37.84 & 37.84 & 18.92 \\
\hline 11. Favorece el uso de otros idiomas & 13.89 & 13.89 & 41.67 & 44.44 \\
\hline 12. No colabora en la mejora de la relación entre los miembros de la Comunidad Educativa & 50 & 38.23 & 11.76 & 0 \\
\hline 13. Con Internet se pierde la relación profesor/a-alumno/a & 43.24 & 43.24 & 13.51 & 0 \\
\hline 14. Con el uso de Internet se pierde la relación entre compañeros/as & 43.24 & 40.59 & 16.22 & 0 \\
\hline 15. El uso de Internet ayuda a hacer nuevos amigos/as & 2.94 & 17.65 & 52.94 & 26.47 \\
\hline 16. El uso de Internet aísla a las personas de su entorno & 11.11 & 44.44 & 38.89 & 5.55 \\
\hline 17. Las TIC facilitan el proceso educativo & 2.78 & 5.55 & 30.55 & 61.11 \\
\hline $\begin{array}{l}\text { 18. El visionado de vídeos a través de Internet resulta muy útil para adquirir nuevos conocimientos, } \\
\text { procedimientos y actitudes }\end{array}$ & 0 & 8.11 & 35.13 & 56.76 \\
\hline 19. El cineforum es un recurso pedagógico interesante, motivador y facilitador de aprendizajes más activos & s 0 & 8.82 & 47.05 & 44.12 \\
\hline 20. Los medios audiovisuales como el cine permiten educar en valores como la solidaridad & 0 & 11.11 & 52.78 & 36.11 \\
\hline $\begin{array}{l}\text { 21. Las TIC se pueden emplear para educar en la diversidad y apoyar a colectivos desfavorecidos socio- } \\
\text { culturalmente }\end{array}$ & 2.78 & 5.56 & 52.78 & 38.89 \\
\hline 22. Internet se puede usar como un instrumento didáctico y no solo de apoyo o refuerzo del libro de texto & 2.78 & 5.56 & 47.22 & 44.44 \\
\hline 23. El uso de las TIC ayuda a abordar de forma eficaz el desarrollo y organización de contenidos & 2.70 & 10.81 & 62.16 & 24.32 \\
\hline 24. El uso de las TIC puede generar nuevos modos de modos de participación y comunicación más activos & & & & \\
\hline y dinámicos & 0 & 12.12 & 39.39 & 48.48 \\
\hline 25. El uso de la plataforma moodle facilita el proceso educativo & 0 & 5.71 & 51.43 & 42.86 \\
\hline 26. Los weblogs facilitarían la comunicación entre el alumnado y con el profesorado & 0 & 14.28 & 45.71 & 40 \\
\hline 27. El uso de wikis permite aprender y compartir contenidos & 0 & 11.43 & 25.71 & 62.86 \\
\hline 28. Los weblogs facilitan las interacciones sociales y el intercambio de opiniones & 0 & 5.88 & 44.12 & 50 \\
\hline 29. El uso de blogs facilitaría la información sobre noticias, temas de interés y eventos relacionados & & & & \\
\hline con la asignatura & 0 & 5.55 & 38.88 & 55.55 \\
\hline 30. Los foros facilitan el intercambio dinámico de información & 2.94 & 5.88 & 41.18 & 50 \\
\hline 31. La tutoría online permite la resolución de dudas facilitando el proceso de enseñanza/aprendizaje & 0 & 17.64 & 52.94 & 29.41 \\
\hline 32. Los cuestionarios en campus virtual ayudan a clarificar y asimilar los contenidos & 0 & 26.47 & 47.06 & 26.47 \\
\hline 33. La exposición de trabajos utilizando Power Point facilita el proceso de aprendizaje & 0 & 17.14 & 45.71 & 37.14 \\
\hline
\end{tabular}

más favorables hacia el uso de las TIC en el proceso de enseñanza/ aprendizaje obteniendo puntuaciones superiores en los ítems 4, 6, 7, 18, 24 y 27 referidos a que Internet puede sustituir la función de los libros y de las pizarras; al hecho de que sería más divertido, útil y provechoso aprender a través de Internet; a que se transmiten valores positivos en Internet; el uso de las TIC puede generar nuevos modos de participación y comunicación más activos y dinámicos; y a que el uso de wikis permite optimizar el proceso de aprendizaje y compartir contenidos. En cambio, obtienen puntuaciones medias inferiores estadísticamente significativas en comparación con la especialidad Lengua y Literatura en los ítems 12, 13 y 14 referidos respectivamente a que el uso de Internet no necesariamente mejora la relación entre los miembros de la Comunidad Educativa; con su uso se pierde más la relación directa entre el profesorado y el alumnado; y también se puede perder la relación y el contacto más personal entre compañeros.

\section{Conclusiones y discusión}

Con respecto al primer apartado de este estudio, como es realizar una evaluación precisa de actitudes e intereses hacia las TIC por estudiantes de posgrado del Máster de Secundaria, los datos obtenidos demuestran que todo el alumnado, estudiantes de postgrado del reciente Máster en Profesorado de Educación Secundaria Obligatoria y Bachillerato, Formación Profesional y Enseñanza de Idiomas de la Universidad de Málaga, conoce Internet y que lo han conocido a través de sus amigos. De mayor importancia resulta el hecho de que la mayoría considera que Internet puede ser empleado como recurso didáctico y no solo como apoyo o refuerzo del libro de texto. A la mayoría del alumnado y del profesorado le gusta el uso de las TIC y suelen considerarlas como herramientas útiles en sus estudios.

En términos generales, los estudiantes de posgrado muestran una actitud muy positiva hacia las TIC tanto 
Tabla 4. Diferencia de medias en percepción de las funciones de las TIC en el contexto educativo en función del género en la especialidad Lengua Extranjera

\begin{tabular}{|c|c|c|c|}
\hline & Hombres & Mujeres & $g l=65$ \\
\hline Ítem & Media $(D T)$ & Media $(D T)$ & $t$ \\
\hline 1. Internet se puede usar para enseñar & $3.00(1)$ & $3.26(.88)$ & -.56 \\
\hline 2. Internet puede sustituir la función del profesor & $2.00(.70)$ & $1.53(.74)$ & 1.38 \\
\hline 3. Internet sólo saben utilizarlo los alumnos con mayor nivel intelectual & $1.20(.45)$ & $1.60(.91)$ & -.93 \\
\hline 4. Internet puede sustituir la función de los libros y las pizarras & $2.60(.55)$ & $2.80(.77)$ & -.53 \\
\hline 5. Internet puede compaginarse con las explicaciones que el profesor realiza en pizarra & $3.40(.55)$ & $3.47(.64)$ & -.21 \\
\hline 6. Sería más útil, divertido y provechoso aprender a través de Internet & $3.20(.84)$ & $2.60(.63)$ & 1.70 \\
\hline 7. Se transmiten valores positivos en Internet & $3.00(.70)$ & $2.73(.80)$ & .66 \\
\hline 8. Internet solo puede utilizarse actualmente en zonas socioeconómicamente favorecidas & $1.80(.84)$ & $2.53(.83)$ & -1.70 \\
\hline 9. El nivel socioeconómico de las familias influye en que conozcamos Internet & $1.60(.55)$ & $2.60(.74)$ & $-2.77 * *$ \\
\hline 10. Internet favorece la capacidad para relacionarse con los demás & $2.00(.70)$ & $2.60(.99)$ & -1.25 \\
\hline 11. Favorece el uso de otros idiomas & $2.80(.84)$ & $3.00(.84)$ & -.46 \\
\hline 12. No colabora en la mejora de la relación entre los miembros de la Comunidad Educativa & $2.40(1.34)$ & $1.73(.70)$ & 1.46 \\
\hline 13. Con Internet se pierde la relación profesor/a-alumno/a & $1.60(.55)$ & $1.93(.96)$ & -.73 \\
\hline 14. Con el uso de Internet se pierde la relación entre compañeros/as & $2.00(1.00)$ & $1.67(.62)$ & .90 \\
\hline 15. El uso de Internet ayuda a hacer nuevos amigos/as & $2.20(.84)$ & $2.93(.96)$ & -1.52 \\
\hline 16. El uso de Internet aísla a las personas de su entorno & $2.40(.89)$ & $2.73(.80)$ & -.79 \\
\hline 17. Las TIC facilitan el proceso educativo & $3.20(.84)$ & $3.40(.74)$ & -.51 \\
\hline $\begin{array}{l}\text { 18. El visionado de vídeos a través de Internet resulta muy útil para adquirir nuevos conocimientos, } \\
\text { procedimientos y actitudes }\end{array}$ & $3.40(.55)$ & $3.26(.70)$ & .38 \\
\hline $\begin{array}{l}\text { 19. El cineforum es un recurso pedagógico interesante, motivador y facilitador de aprendizajes } \\
\text { más activos }\end{array}$ & $3.60(.55)$ & $3.06(.70)$ & 1.54 \\
\hline 20. Los medios audiovisuales como el cine permiten educar en valores como la solidaridad & $3.20(.45)$ & $3.00(.65)$ & .63 \\
\hline $\begin{array}{l}\text { 21. Las TIC se pueden emplear para educar en la diversidad y apoyar a colectivos desfavorecidos } \\
\text { socioculturalmente }\end{array}$ & $3.20(.45)$ & $3.06(.59)$ & .46 \\
\hline 22. Internet se puede usar como un instrumento didáctico y no solo de apoyo o refuerzo del libro & & & \\
\hline de texto & $3.20(.45)$ & $3.33(.49)$ & -.54 \\
\hline 23. El uso de las TIC ayuda a abordar de forma eficaz el desarrollo y organización de contenidos & $3.20(.45)$ & $2.93(.59)$ & .92 \\
\hline $\begin{array}{l}\text { 24. El uso de las TIC puede generar nuevos modos de modos de participación y comunicación más } \\
\text { activos y dinámicos }\end{array}$ & $3.40(.55)$ & 3.40 & .00 \\
\hline 25. El uso de la plataforma moodle facilita el proceso educativo & $3.40(.55)$ & $3.06(.88)$ & .79 \\
\hline 26. Los weblogs facilitarían la comunicación entre el alumnado y con el profesorado & $3.80(.44)$ & $3.07(.70)$ & $2.17 *$ \\
\hline 27. El uso de wikis permite aprender y compartir contenidos & $3.60(.55)$ & $3.53(.64)$ & .21 \\
\hline 28. Los weblogs facilitan las interacciones sociales y el intercambio de opiniones & $3.60(.55)$ & $3.40(.63)$ & .63 \\
\hline $\begin{array}{l}\text { 29. El uso de blogs facilitaría la información sobre noticias, temas de interés y eventos relacionados } \\
\text { con la asignatura }\end{array}$ & $3.60(.55)$ & $3.33(.62)$ & .86 \\
\hline 30. Los foros facilitan el intercambio dinámico de información & $3.40(.89)$ & $3.28(.61)$ & .32 \\
\hline 31. La tutoría online permite la resolución de dudas facilitando el proceso de enseñanza/aprendizaje & $3.60(.55)$ & $3.07(.70)$ & 1.54 \\
\hline 32. Los cuestionarios en campus virtual ayudan a clarificar y asimilar los contenidos & $3.20(.84)$ & $2.87(.64)$ & .94 \\
\hline 33. La exposición de trabajos utilizando Power Point facilita el proceso de aprendizaje & $3.20(.45)$ & $3.13(.74)$ & .19 \\
\hline
\end{tabular}

Significación estadística. ${ }^{*} p<0.05 ; * *<0.0$

como herramienta de comunicación (con un claro predominio del uso del correo electrónico) así como para actividades relacionadas con la búsqueda, transmisión y presentación de la información. Estos resultados son congruentes con otros estudios que muestran un uso cada vez más generalizado de estas herramientas como recursos especialmente motivante y relevantes para el alumnado (Martínez y Aguaded, 2004). Además la mayoría del alumnado considera que las TIC favorecen el uso de otros idiomas. De hecho, cada vez se emplean más tanto para animar a la lectura como para el aprendizaje de idiomas en numerosos centros de estudio. Para finalizar, puede destacarse que, la mayoría están poco o nada de acuerdo con la afirmación de que
Internet puede sustituir la función del profesorado y consideran que las TIC pueden emplearse para educar en la diversidad; ambos aspectos son de interés con vistas a una educación integral dirigida a la mejora de la convivencia social. De hecho, algunos autores (Javaloy, Espelt y Cornejo, 2001) encuentran que estos recursos contribuyen a potenciar el denominado paradigma de la identidad que enfatiza aspectos sociales tan necesarios como la conciencia social y los valores y de ahí la necesidad de su empleo también con esta finalidad.

El alumnado de posgrado del reciente Máster de Educación Secundaria participante en este estudio ha otorgado importancia a la utilización del visionado de 
Tabla 5. Diferencia de medias en percepción de las funciones de las TIC según la especialidad

\begin{tabular}{|c|c|c|c|}
\hline & $\begin{array}{l}\text { Lengua } \\
\text { Extranjera }\end{array}$ & $\begin{array}{l}\text { Lengua, } \\
\text { Latín y Griego }\end{array}$ & $g l=177$ \\
\hline Ítem & Media $(D T)$ & Media $(D T)$ & $t$ \\
\hline 1. Internet se puede usar para enseñar & $3.42(.81)$ & $3.34(.54)$ & .46 \\
\hline 2. Internet puede sustituir la función del profesor & $1.64(.68)$ & $1.37(.49)$ & 1.90 \\
\hline 3. Internet sólo saben utilizarlo los alumnos con mayor nivel intelectual & $1.42(.69)$ & $1.31(.53)$ & .70 \\
\hline 4. Internet puede sustituir la función de los libros y las pizarras & $2.53(.88)$ & $1.94(.64)$ & $3.22 * *$ \\
\hline 5. Internet puede compaginarse con las explicaciones que el profesor realiza en pizarra & $3.56(.69)$ & $3.37(.65)$ & 1.16 \\
\hline 6. Sería más útil, divertido y provechoso aprender a través de Internet & $2.92(.69)$ & $2.40(.77)$ & $2.98 * *$ \\
\hline 7. Se transmiten valores positivos en Internet & $2.75(.69)$ & $2.38(.55)$ & $2.45^{*}$ \\
\hline 8. Internet solo puede utilizarse actualmente en zonas socioeconómicamente favorecidas & $2.03(.88)$ & $2.29(.98)$ & -1.16 \\
\hline 9. El nivel socioeconómico de las familias influye en que conozcamos Internet & $2.39(.84)$ & $2.60(.95)$ & -1.00 \\
\hline 10. Internet favorece la capacidad para relacionarse con los demás & $2.58(.91)$ & $2.31(.96)$ & 1.21 \\
\hline 11. Favorece el uso de otros idiomas & $3.11(.82)$ & $2.97(.74)$ & .75 \\
\hline 12. No colabora en la mejora de la relación entre los miembros de la Comunidad Educativa & $1.74(.83)$ & $2.26(.74)$ & $-2.76^{* *}$ \\
\hline 13. Con Internet se pierde la relación profesor/a-alumno/a & $1.81(.79)$ & $2.38(.95)$ & $-2.77 * *$ \\
\hline 14. Con el uso de Internet se pierde la relación entre compañeros/as & $1.78(.72)$ & $2.17(.86)$ & $-2.10^{*}$ \\
\hline 15. El uso de Internet ayuda a hacer nuevos amigos/as & $2.86(.87)$ & $2.71(.75)$ & .76 \\
\hline 16. El uso de Internet aísla a las personas de su entorno & $2.50(.77)$ & $2.71(.79)$ & -1.10 \\
\hline 17. Las TIC facilitan el proceso educativo & $3.42(.77)$ & $3.15(.78)$ & 1.45 \\
\hline $\begin{array}{l}\text { 18. El visionado de vídeos a través de Internet resulta muy útil para adquirir nuevos conocimientos, } \\
\text { procedimientos y actitudes }\end{array}$ & $3.50(.61)$ & $3.11(.76)$ & $2.37 *$ \\
\hline $\begin{array}{l}\text { 19. El cineforum es un recurso pedagógico interesante, motivador y facilitador de aprendizajes } \\
\text { más activos }\end{array}$ & $3.38(.65)$ & $3.22(.69)$ & .95 \\
\hline 20. Los medios audiovisuales como el cine permiten educar en valores como la solidaridad & $3.25(.65)$ & $3.00(.77)$ & 1.48 \\
\hline $\begin{array}{l}\text { 21. Las TIC se pueden emplear para educar en la diversidad y apoyar a colectivos desfavorecidos } \\
\text { socioculturalmente }\end{array}$ & $3.28(.70)$ & $3.14(.69)$ & .82 \\
\hline 22. Internet se puede usar como un instrumento didáctico y no solo de apoyo o refuerzo del libro & & & \\
\hline de texto & $3.30(.71)$ & $2.97(.86)$ & 1.79 \\
\hline 23. El uso de las TIC ayuda a abordar de forma eficaz el desarrollo y organización de contenidos & $3.08(.69)$ & $3.06(.72)$ & .16 \\
\hline $\begin{array}{l}\text { 24. El uso de las TIC puede generar nuevos modos de modos de participación y comunicación más } \\
\text { activos y dinámicos }\end{array}$ & $3.47(.65)$ & $3.03(.66)$ & $2.84 *$ \\
\hline 25. El uso de la plataforma moodle facilita el proceso educativo & $3.19(.82)$ & $3.03(.56)$ & .95 \\
\hline 26. Los weblogs facilitarían la comunicación entre el alumnado y con el profesorado & $3.17(.77)$ & $3.00(.54)$ & 1.05 \\
\hline 27. El uso de wikis permite aprender y compartir contenidos & $3.42(.77)$ & $3.03(.74)$ & $2.10 *$ \\
\hline 28. Los weblogs facilitan las interacciones sociales y el intercambio de opiniones & $3.36(.72)$ & $3.18(.57)$ & 1.18 \\
\hline $\begin{array}{l}\text { 29. El uso de blogs facilitaría la información sobre noticias, temas de interés y eventos relacionados } \\
\text { con la asignatura }\end{array}$ & $3.39(.73)$ & $3.29(.46)$ & .72 \\
\hline 30. Los foros facilitan el intercambio dinámico de información & $3.29(.82)$ & $3.06(.55)$ & 1.35 \\
\hline 31. La tutoría online permite la resolución de dudas facilitando el proceso de enseñanza/aprendizaje & $3.06(.75)$ & $2.91(.92)$ & .71 \\
\hline 32. Los cuestionarios en campus virtual ayudan a clarificar y asimilar los contenidos & $2.95(.79)$ & $2.83(.75)$ & .63 \\
\hline 33. La exposición de trabajos utilizando Power Point facilita el proceso de aprendizaje & $3.19(.71)$ & $2.86(.76)$ & 1.77 \\
\hline
\end{tabular}

Significación estadística. ${ }^{*} \mathrm{p}<0.05 ; * * \mathrm{p}<0.01$

vídeo y del cineforum en su proceso de aprendizaje. En este sentido, según pone de manifiesto Bartolomé (2008), la digitalización del vídeo representa una nueva oportunidad para avanzar en la alfabetización mediática, para potenciar las redes sociales y los aprendizajes colaborativos con ayudas del audiovisual, y convertir las sesiones de clase en espacio de encuentro, intercambio y debate. Otro estudio (Pereira, 2003) argumenta la necesidad de servirse de los medios audiovisuales como el cine incluso para abordar de una forma viva temas transversales y relevantes como la educación en valores como la solidaridad.

Puede destacarse que los estudiantes de posgrado participantes en este estudio tienen, por tanto, muy buena predisposición hacia el uso de las TIC en su proceso de aprendizaje. También otorgan bastante importancia al uso de herramientas colaborativas de la Web 2.0 (foros, wikis, chat, blogs, redes sociales) para su formación en competencias. Este resultado guarda coherencia con otros estudios (Fernández y Mena, 2010; Ríos y Ruiz, 2011) que exhiben que el uso de estas herramientas tiene un gran potencias en el campo de la educación, tanto en el aprendizaje formal como informal, ya que a través de ellas el alumnado puede expresarse por sí mismo a la vez que se promueven relaciones y otros aspectos relacionados con la comunicación con otros miembros de la comunidad educativa y de fuera de ella. 
En la misma línea que los estudios anteriores, según se desprende de los datos aportados por el Instituto Nacional de Tecnologías de la Comunicación en el año 2009 el 69\% de los usuarios de redes sociales en España correspondía a la franja de edad de los 15 a los 34 años, por lo que es importante considerar sus potencialidades también en la educación. De hecho, según Castañeda (2010) es necesario hablar de las redes sociales en relación con la educación desde tres perspectivas educativas complementarias: 1) Aprender con redes sociales (aprendizajes que se llevan a cabo dentro de la educación formal y no formal o lo que es lo mismo, aprendizajes reglados); 2) Aprender a través de redes sociales (procesos de aprendizaje informales en los que los usuarios de las redes pueden aprender de manera autónoma e independiente al formar parte de esa red); y 3) Aprender a vivir en un mundo de redes sociales (necesidad de informar y concienciar de que el uso que se haga de ellas ampliará las posibilidades que ofrecen así como del papel de las mismas en toda nuestra vida en los diferentes roles, como aprendices y como personas, que se es capaz de asumir). Se puede señalar que las redes sociales se han introducido de forma significativa en nuestras vidas y la educación no puede permanecer ajena a esta realidad.

Respecto al segundo apartado, cuyo objetivo general es analizar diferencias en la predisposición y actitudes hacia las TIC en función del género y de la especialidad de dicho Máster, los resultados obtenidos en este estudio demuestran que existen diferencias en la percepción de las funciones de las TIC en el contexto educativo universitario y en la predisposición hacia las mismas en función del género siendo los hombres de la especialidad Lengua Extranjera los que consideran en mayor medida que los weblogs facilitarían la comunicación entre el alumnado y con el profesorado y otorgan menos importancia que las mujeres al ítem referido a que el nivel socieconómico de las familias puede influir en que conozcamos Internet. Estos resultados son congruentes con otras investigaciones (Wallace y Clariana, 2005) que consideran a la variable género como relevante en este tipo de estudios. No obstante, se puede manifestar que tan solo en dichos aspectos relacionados con las TIC se han obtenido diferencias significativas. Este último dato también es coherente con otros estudios que no encuentran diferencias estadísticamente significativas entre hombres y mujeres (Adebanjo, 2004; Hoxmier, Nie y Purvis, 2000; Tekinarslan, 2008) en actitudes hacia las TIC y otros aspectos relacionados con el conocimiento y uso de los ordenadores. Asimismo, también se han encontrado diferencias estadísticamente significativas en lo que a la percepción y actitudes hacia las TIC en función de la especialidad se refiere. Como resultados más significa- tivos, se encuentra que los estudiantes de postgrado pertenecientes a la especialidad Lengua Extranjera muestran mayor predisposición hacia el uso de las TIC en el contexto educativo universitario en comparación con los de la especialidad Lengua, Literatura, Latín y Griego. No obstante, se están analizando otros datos como la predisposición de los docentes de dicho Máster hacia las TIC y se hace necesario seguir profundizando en futuras investigaciones en dichas variables para obtener resultados más concluyentes al respecto.

Para finalizar, se señala que la información obtenida resulta de interés tanto para la formación de los docentes como para la integración de las TIC en el currículo académico. Se aportan datos relevantes que pueden contribuir a optimizar el proceso de enseñanza/aprendizaje en el reciente Máster en Profesorado de Educación Secundaria Obligatoria y Bachillerato, Formación Profesional y Enseñanza de Idiomas celebrado en estos primeros cursos académicos en la Universidad de Málaga. Ante las continuas transformaciones que están experimentando las universidades al igual que el resto de la sociedad, el mundo educativo no puede quedarse fuera de las muchas posibilidades de aplicación que brindan las tecnologías digitales en la educación potenciando el desarrollo de numerosos conocimientos, procedimientos y aptitudes transversales como la competencia social, las aptitudes de comunicación y organización las posibilidades formadoras de los docentes y el alumnado. El fomento de dichas competencias relacionadas con las TIC hace más fácil contactar con realidades muy diferentes y contribuye al enriquecimiento personal del alumnado y del profesorado; eliminando o disminuyendo barreras para el acceso a los recursos y oportunidades que brinda la educación superior, facilitando el desarrollo de planteamientos más colaborativos con el aprendizaje, y favoreciendo el aprendizaje autónomo así como el seguimiento de nuevos enfoques a los habituales de la enseñanza y aprendizaje tradicional que, cuando menos, complementan estos últimos. También permitiría proporcionar, entre otras muchas ventajas, un feedback más efectivo y el intercambio continuo de información, aspectos ambos tan necesarios en el ámbito de la formación y de la educación.

\section{Agradecimientos}

Agradecer al alumnado del Máster en Profesorado de Educación Secundaria Obligatoria y Bachillerato, Formación Profesional y Enseñanza de Idiomas de la Universidad de Málaga su buena disposición para responder a la las cuestiones planteadas en este estudio. 


\section{Extended Summary}

The New Model for Higher Education brings new methodologies, demands and challenges, in which the use of ICT, especially the internet, as a teaching tool, will be indispensable in teaching and learning.

In the process of European convergence, young people must be prepared in skills to enable them to cope as successful professionals, that is, highly qualified individuals who will guide the economic, intellectual and cultural needs of our societies. To promote the acquisition of these skills, collaborative, participatory methodologies are needed, such as those offered by Web 2.0. In this sense, Fernandez and Mena (2010) consider the role of the teacher and the concept of teaching to have changed, as professionals must now develop new skills, both personal and professional, such as the use of ICT tools, initiative, creativity, teamwork and problem-solving strategies, to gain the necessary knowledge to enable them to successfully face the challenge of assuming the new role of mediator, and which foster teamwork (or collaborative) as well as independent (or critical) thinking.

At the university level, some studies (De Pablos, 2009, Trujillo, 2005) have stressed the need not to use ICT indiscriminately without reflecting on the introduction these changes involve, considering such aspects as their degree of usefulness and adaptability. In this sense, an important element in the process of integration of ICT at the University is to know precisely the views and attitudes of students regarding the use of ICTs in their learning process (Martinez and Aguaded, 2004).

Specifically, the objective of this study is to provide an assessment of attitudes and interests towards ICTs in a sample of 224 graduate students studying for the Master's degree in Teaching Secondary Education, specializing in foreign languages (English and French), Latin and Greek language and literature, and Technology, Computer Science and Industrial Processes. Differences were analyzed according to gender and specialty.

The participants were 224 students, aged between 22 and 49, at the University of Malaga, with 64 specializing in English and French as foreign languages, 58 specializing in Latin and Greek language and literature, and 102 taking part in two courses (morning and afternoon) in the specialty of TIPI (Technology, Information Technology and Industrial Processes).

This research used the Questionnaire of Attitudes and Interests towards ICTs (Morales, 2009). The questions included refer to the level of knowledge about computers, their frequency of use, the way in which the internet is accessed and to what extent it useful to use videos, PowerPoint, Moodle, forums, wikis, chats, and Video Forums blogs in the learning process, as well as issues related to other possible features and applications of the internet in the context of university education. The format of the questionnaire is a series of questions which participants are asked to complete, or to circle the option that corresponds to their answer. For functions that the internet can have in the educational context, students were presented with a Likert scale to state their level of agreement with 15 statements, where $0=$ not at all, $1=$ slightly, $2=$ fairly, $3=$ very much. This questionnaire has adequate psychometric properties.

With regard to the procedure, it is worth noting that participants filled out the questionnaire on a voluntary basis. The data were then entered into SPSS for further analysis. The assessment instrument was administered to participants collectively, in class time, on a date and at a time determined by the University's academic calendar. The administration of the questionnaire was conducted by psychologists who were experts in the field. The coding and analysis was done with SPSS 15.0. In addition to descriptive analysis reflecting percentages, means and standard deviations, we performed Student's $t$-test for independent samples to see if there were significant differences according to gender and the specific specialty of the Master's in Teaching Secondary Education. Specifically, this study compares results regarding the attitudes and interests towards ICT between specialties in English and French as a Foreign Language, Latin and Greek language and literature, and Technology, Computer and Industrial Processes.

The results show that all students who graduate know what the internet is $(100 \%)$. When asked how they knew about it, $39.02 \%$ of respondents claim that they first encountered it through friends, $21.95 \%$ through school and through the family, television or other means. All participants have a personal computer, most of them access the internet from home $(48.44 \%)$ with a frequency of one or more times a day and have no website. The internet services most used when accessing the network are, in order of use: mail (26.89\%), search engines (26.89\%), forums $(21.85 \%)$, chats $(12.60 \%)$ or other services like social networks $(11.76 \%) .100 \%$ of students feel that the internet can be addictive. $100 \%$ of the participants in this study believe that the internet could be used as a teaching resource. With regard to the extent that students like the internet, the students gave an average score of 9.21 (on a scale of 0-10).

The participants claim, generally speaking, that their computer skills are at the user level in terms of databases and spreadsheets, while claiming to have less knowledge about programming.

With respect to the statistically significant differences in the perception of the role of ICTs in the context of university according to gender, it can be noted that women place a greater emphasis than men on the socioeconomic status of families for knowledge about 
the internet, while men place greater importance on the fact that weblogs facilitate communication between students and faculty.

With regard to the specialty, the results show no statistically significant differences in the predisposition towards ICT between students in the specialty of foreign languages and that of Technology, Computers and Industrial Processes. However, there are significant differences in perception and bias towards ICT between students in the specialty of Foreign Languages and the specialty of Latin and Greek Language and Literature.

Students in the Foreign Language specialty show more willingness and more favorable attitudes toward the use of ICTs in the teaching / learning process, and obtained higher scores on items 4, 6, 7, 18, 24 and 2 . These items refer to whether the internet can replace the role of books and boards, whether it would be more fun, useful and helpful to learn via the internet, whether positive values are transmitted on the internet, whether the use of ICTs can create new, active and dynamic modes of participation and communication, and whether wikis can be used to optimize the learning process and share content. However, in comparison, students in the language and literature specialty believe that internet use does not necessarily improve the relationship between members of the education community, and that it may cause the relationship between teachers and students to be lost, as well as the personal contact with colleagues.

Overall, graduate students show a very positive attitude towards ICTs, both as a communication tool (with a clear predominance of the use of electronic mail), as well as for activities related to searching for, and the transmission and presentation of, information. These results are consistent with other studies showing an increasingly widespread use of these tools as resources which are especially relevant and motivating for students (Martinez and Aguaded, 2004).

It may be noted that the graduate students who participated in this study, are, as such, well disposed towards the use of ICTs in the learning process. In this sense, importance is given to the use of the collaborative tools of Web 2.0 (forums, wikis, chat, blogs, social networks) for skills training. This result is consistent with other studies (Fernandez and Mena, 2010; Rivers and Ruiz, 2011) that show that the use of these tools has great powers in the field of education, in both formal and informal learning, since through them students can express themselves while developing interpersonal relationships, and other aspects related to communication, with other members of the educational community and beyond.

These graduate students consider the use of video and Cine Forum to be important in the learning process. Indeed, as evidenced by Bartholomew (2008), video digitization represents a new opportunity to advance media literacy, to enhance social networks and collaborative learning with audiovisual aids, and make the class sessions a meeting space for exchange and debate.

These data allow us to reaffirm the importance of using ICTs in the teaching / learning process, as well as the possible advantages and drawbacks of its use by both the faculty and graduate students of the recent Master's in Secondary Education.

\section{Referencias}

Adebanjo, A. A. (2004). The attitude and gender difference in the utilization of computer among undergraduates. Journal of Computer Literacy, 5, 171-183.

Barroso, J. y Cabero, J. (2010). La investigación educativa en TIC. Visiones prácticas. Madrid: Síntesis.

Bartolomé, A. (2008). Vídeo digital y educación. Madrid: Síntesis.

Cabero, J. y Gisbert, M. (2005). Formación en Internet. Guía para el diseño de materiales didácticos. Sevilla: MAD.

Carr, N. (2011). Aguas superficiales. ¿Qué está haciendo Internet con nuestras mentes? Madrid: Taurus.

Castañeda, L. (2010). Aprendizaje con redes sociales. Tejidos educativos para los nuevos entornos. Sevilla: MAD.

Cebrián, M. y Gallego, M. J. (2011). Procesos educativos con TIC en la sociedad del conocimiento. Madrid: Pirámide.

De Pablos, J. (2009). Tecnología educativa. La formación del profesorado en la era de Internet. Málaga: Aljibe.

De Pablos, J., Area, M., Valverde, J. y Correa, J. M. (2010). Políticas educativas y buenas prácticas con TIC. Barcelona: GRAÓ.

Fernández, M. A. y Mena, E. (2010). El tutor 2.0: Aplicaciones para entornos virtuales de aprendizaje. Málaga: Aljibe.

García-Valcárcel, A. (2007). Herramientas tecnológicas para mejorar la docencia universitaria. Una reflexión desde la experiencia y la investigación. Revista Iberoamericana de Educación a Distancia, 10, 125-148.

Hoxmier, J. A., Nie, W., y Purvis, G. T. (2000). The impact of gender and experience on user confidence in electronic mail: Industry trend or event. Journal of End User Computing, 7, 99-106.

Instituto Nacional de Tecnologías de la Comunicación (2009). Estudio sobre la privacidad de los datos personales y la seguridad de la información en las redes sociales online (documento en línea). Extraído de www.inteco.es el 2 de junio de 2010.

Javaloy, F., Espelt, E. y Cornejo, J. M. (2001). Internet y movimientos sociales: un enfoque psicosocial. Anuario de Psicología, 32, 31-37.

Martínez, T. y Aguaded, J. (2004). El uso de las TIC en alumnos principiantes de las universidades españolas. Granada: GEU.

Morales, F. M. (2009, diciembre). Empleo de TIC en la enseñanza práctica: Análisis comparativo en diferentes titula- 
ciones universitarias. Comunicación presentada en el I Congreso Internacional sobre uso y buenas prácticas con TIC, Universidad de Málaga, España.

Pereira, C. (2003). El cine, nuevo escenario de la educación. En Romañá Blay, M. T. y Martínez Martín, M. (Eds.), Otros lenguajes en educación. Barcelona: ICEUniversitat de Barcelona, pp. 101-105.

Ríos, J. M. y Ruiz, J. (2011). Competencias, TIC e innovación. Nuevos escenarios para nuevos retos. Sevilla: MAD.

Rubio, A. y Álvarez, A. (2010). Formación de formadores después de Bolonia. Madrid: Díaz de Santos.
Sangrá A. y González, M. (2004). La transformación de las universidades a través de las TIC: discursos y prácticas. Barcelona: UOC.

Tekinarslan, E. (2008). Computer anxiety: A cross-cultural study of Dutch and Turkish University students. Computers in Human Behaviour, 24, 1572-1584.

Trujillo, A. (2005). Nuevas tecnologías y psicología. Una perspectiva actual. Apuntes de Psicología, 23, 321335.

Wallace, P. y Clariana, R. B. (2005). Gender difference in computer-administered versions Paper-based Tests. International Journal of Instructional Media, 32, 37-45.

Manuscrito recibido: 30/12/2011

Revisión recibida: 22/02/2012

Manuscrito aceptado: 27/02/2012 